\title{
Comparison of a Free-Breathing CT and an Expiratory Breath-Hold CT with Regard to Spatial Alignment of Amplitude-Based Respiratory-Gated PET and CT Images
}

\author{
Charlotte S. van der Vos ${ }^{1,2}$, Willem Grootjans ${ }^{1}$, Antoi P.W. Meeuwis ${ }^{1}$, Cornelis H. Slump ${ }^{2}$, Wim J.G. Oyen ${ }^{1}$, \\ Lioe-Fee F. de Geus-Oei ${ }^{1,2}$, and Eric P. Visser ${ }^{1}$ \\ ${ }^{I}$ Department of Radiology and Nuclear Medicine, Radboud university medical centre, Nijmegen, The Netherlands; \\ and ${ }^{2}$ MIRA Institute for Biomedical Technology and Technical Medicine, University of Twente, Enschede, The Netherlands
}

Respiratory motion during PET has a significant effect on the quantification of radiotracer uptake in PET images. Even when respiratory motion is considered using PET gating techniques, inaccuracies in standardized uptake values can be caused by inappropriate attenuation correction due to a spatial mismatch between PET and CT. In this study, the effect of breath-hold CT imaging on the spatial match between CT and amplitude-based respiratory-gated PET images is investigated. Methods: Wholebody ${ }^{18} \mathrm{~F}-\mathrm{FDG} \mathrm{PET} / \mathrm{CT}$ imaging was performed in 52 patients with 125 lung lesions. ${ }^{18}$ F-FDG PET was performed using optimized, amplitude-based respiratory gating. For CT, 36 patients were randomly assigned to the free-breathing (FB) group and 16 to the restexpiratory breath-hold $(\mathrm{BH})$ group. Spatial mismatch between the PET and CT images was quantified by measuring the distance between the centroids of PET and CT lesions and calculating the Jaccard similarity coefficient (JSC). Results: In the upper lobes, the average distance between the centroids of the PET and CT lesions was $4.7 \pm 3.1$ and $6.0 \pm 3.0 \mathrm{~mm}$ for the FB and $\mathrm{BH}$ groups, respectively $(P=0.11)$. For the middle and lower lobes, the distances were $5.8 \pm 4.3$ and $5.1 \pm 2.9 \mathrm{~mm}(P=0.70)$, respectively, and for the central region $4.8 \pm 4.6$ and $5.6 \pm 2.0 \mathrm{~mm}$ $(P=0.24)$, respectively. The JSC for the upper lobes was $0.28 \pm$ 0.17 and $0.28 \pm 0.19$, for the $\mathrm{FB}$ and the $\mathrm{BH}$ group, respectively $(P=0.83)$. For the middle and lower lobes, the JSC was $0.22 \pm$ 0.16 and $0.28 \pm 0.18(P=0.20)$, respectively, and for the central region $0.39 \pm 0.17$ and $0.13 \pm 0.04(P=0.04)$, respectively. Conclusion: Providing breathing instructions to the patients during the CT acquisition did not improve the spatial alignment between the respiratory-gated PET images and the CT images. The difficulty experienced in using this clinical protocol, such as patient compliance and operator dependence, emphasizes the need for other strategies.

Key Words: amplitude-based optimal respiratory gating; lung tumors; standardized uptake value; breath hold CT; spatial alignment

J Nucl Med Technol 2014; 42:269-273

DOI: 10.2967/jnmt.114.145748

Received Jul. 21, 2014; revision accepted Sep. 23, 2014.

For correspondence or reprints contact: Charlotte S. van der Vos, Department of Radiology and Nuclear Medicine, Radboud University Medical Centre, P.O.

Box 9101, 6500 HB Nijmegen, The Netherlands.

E-mail: charlotte.vandervos@radboudumc.nl

Published online Oct. 23, 2014.

COPYRIGHT $\odot 2014$ by the Society of Nuclear Medicine and Molecular Imaging, Inc.
$\mathbf{P}$ molecular imaging method for accurate staging and diagnosis of a variety of diseases, particularly in oncology $(1,2)$. The advantage of combined PET/CT imaging is that it provides both anatomic and molecular information on the patient, improving detection, localization, and characterization of disease (2). Furthermore, the CT scan can be used for PET attenuation correction. Quantitative indices in PET, such as the standardized uptake value (SUV) (3), metabolic volume, and total lesion glycolysis $(4,5)$, can be used to diagnose the disease, to provide prognostic and predictive information, and to optimize radiotherapy planning. Furthermore, it has been established that molecular imaging with PET is valuable in the early assessment of therapy response of several tumor types (6-9).

During PET acquisition, patients are instructed to breathe freely because of the relatively long image acquisition times. As a consequence, respiratory motion can result in significant blurring of structures within the thorax and upper abdomen, reducing quantitative accuracy of radiotracer uptake and accurate volume definition in PET images (10-13). Different strategies have been developed in an attempt to correct PET images for respiratory motion. These methods, collectively known as respiratory gating, have demonstrated that breathing-induced image-blurring can significantly affect the quantification of radiotracer uptake in PET images. Additionally, underestimation and overestimation of the SUVs can also be caused by a spatial mismatch between PET and CT, resulting in inappropriate PET attenuation correction $(14,15)$. The spatial mismatch can even be increased by respiratory gating on the PET images, because the lesions can be captured in a respiratory phase different from the CT scan. The effect of inappropriate attenuation correction on quantification of radiotracer uptake will be largest in moving lesions at the interface of anatomic regions with different densities (e.g., the lower lung and liver dome) (16).

Improving the spatial match between the PET and CT images can therefore result in more accurate attenuation correction and lesion SUVs. Correction for these inaccuracies in SUV may improve the diagnostic accuracy of PET imaging 
TABLE 1

Demographics of Patient Population

\begin{tabular}{|c|c|c|}
\hline Characteristic & FB group & $\mathrm{BH}$ group \\
\hline Average age in $y$ & $66.7(9.5)$ & $70.9(9.8)$ \\
\hline Average weight in $\mathrm{kg}$ & $77.7(12.1)$ & $70.6(11.9)$ \\
\hline \multicolumn{3}{|l|}{ Sex } \\
\hline Female & 12 & 6 \\
\hline Male & 24 & 10 \\
\hline \multicolumn{3}{|l|}{ Confirmed malignancy } \\
\hline Primary lung cancer & 25 & 10 \\
\hline Metastasis & 3 & 3 \\
\hline Other and unconfirmed & 8 & 3 \\
\hline Lesion size on CT in $\mathrm{mm}^{3}$ & $8,261.6(22,914.4)$ & $8,890.2(21,872.8)$ \\
\hline Average ${ }^{18} \mathrm{~F}-\mathrm{FDG}$ dose in $\mathrm{MBq}$ & $247.7(40.2)$ & $225.8(41.5)$ \\
\hline Minimum SUV $V_{\max }$ in $\mathrm{g} / \mathrm{cm}^{3}$ & 0.67 & 1.03 \\
\hline $\begin{array}{l}\text { Data in parentheses are SDs. } \\
\text { SUV }_{\max }=\text { maximum SUV. }\end{array}$ & & \\
\hline
\end{tabular}

and may facilitate, in particular, clinical decision making during early treatment response monitoring and the incorporation of PET in radiotherapy treatment planning (13).

To improve PET/CT coregistration, the change of freebreathing (FB) CT acquisitions into CT acquisition protocols with breathing instructions provided has been suggested $(15,17,18)$. In the present study, the effect of breath-hold (BH) CT imaging on the spatial match between CT and amplitudebased respiratory-gated PET images is investigated.

\section{MATERIALS AND METHODS}

\section{Patients}

Whole-body (WB) ${ }^{18} \mathrm{~F}-\mathrm{FDG}$ PET/CT imaging was performed in 52 patients with 125 lung lesions. The demographics are shown in Table 1 . When respiratory gating became available for ${ }^{18} \mathrm{~F}$-FDG $\mathrm{PET} / \mathrm{CT}$ in our institute, it was included in the routine diagnostic work-up of patients with suspected lung cancer. Patients were scanned with or without respiratory instruction during the CT scan. This study was approved by the institutional review board (IRB) and performed in accordance with the applicable rules concerning the review of research ethics committees, and the requirement to obtain informed consent was waived. The localizations of the lesions used for image analysis are summarized in Table 2 .

\section{Image Acquisition}

Images were acquired using a Siemens Biograph $40 \mathrm{mCT}$ PET/CT scanner with optimized, amplitude-based respiratory gating (HD-Chest) (12). The PET scanner has an extended axial field of view of $216 \mathrm{~mm}$ with 4 lutetium oxyorthosilicate detector rings. A dose of $3.2 \mathrm{MBq}$ of ${ }^{18} \mathrm{~F}-\mathrm{FDG}$ per kilogram of body weight was administered. Respiratory gating was performed on bed positions covering the thorax. Gated and nongated bed positions were scanned during FB for 6 and 2 min, respectively. The respiratory signal was obtained using an Anzai AZ-733V respiratory gating system. This system consists of a pressure sensor integrated in an elastic belt placed around the patient's thorax. A WB low-dose CT scan was acquired for attenuation correction and anatomic reference. The $\mathrm{x}$-ray tube peak voltage $(\mathrm{kVp})$ was set to 100 and $120 \mathrm{kV}$ for patients with body mass less than $70 \mathrm{~kg}$ and greater than $70 \mathrm{~kg}$, respectively. The tube current was modulated using CARE Dose4D (Siemens), with a reference setting of $50 \mathrm{mAs}$. The CT scan was acquired with $5.0 \mathrm{~mm}$ slices $(16 \times 1.2)$, a pitch of 1.0 , and a rotation time of $0.5 \mathrm{~s}$. For CT acquisition, patients were sequentially assigned to 2 groups using different breathing protocols: a FB group $(n=36)$ and a $\mathrm{BH}$ group $(n=16)$. In the FB group, no breathing instructions were given to the patients during $\mathrm{CT}$ imaging. The patients in the $\mathrm{BH}$ group received an end rest-expiratory $\mathrm{BH}$ instruction during CT imaging. Seven patients originally assigned to the $\mathrm{BH}$ group could not comply with the respiratory instruction. These patients were assigned to the FB group.

\section{Image Reconstruction}

The PET images were reconstructed using an algorithm with a spatially varying point-spread function incorporating time-of-flight information (UltraHD PET; Siemens). Images were reconstructed with 3 iterations, 21 subsets, and a transaxial matrix size of $400 \times 400$ (pixel size, $2.04 \times 2.04 \mathrm{~mm}^{2}$ ). The slice thickness of the PET images was matched to the slice thickness of the attenuation CT, and postreconstruction filtering was performed with a 3-dimensinal gaussian filter kernel with a full width at half maximum of $3.0 \mathrm{~mm}$. Respiratory gating was performed on the list-mode data with an amplitude-based optimal respiratory-gated algorithm, integrated in the Syngo 2011A MI.PET/CT software. The main user input for the optimal respiratory-gated algorithm is the percentage duty cycle, which is the percentage of the total number of true coincidences used for image reconstruction. The optimal respiratory-gated algorithm calculates an optimal amplitude range for a given duty cycle (12). Selection of the percentage duty cycle permits the user to control the amount of noise versus the residual motion components in the reconstructed images. In this study, images were reconstructed using a duty cycle of $35 \%$, corresponding to $126 \mathrm{~s}$ of PET data per gated bed position. This duty cycle provides the best trade-off between number of counts, scan time, and reduction of

TABLE 2

Number of Lesions for FB and BH Groups

\begin{tabular}{lcc}
\hline \multicolumn{1}{c}{ Anatomic location } & FB group & BH group \\
\hline Upper lobes & 45 & 17 \\
Middle and lower lobes & 30 & 19 \\
Central & 11 & 3 \\
Total & 86 & 39 \\
\hline
\end{tabular}


motion artifacts $(12,13)$. For the nongated images, the first $126 \mathrm{~s}$ of the acquired data was used for image reconstruction.

The CT images for attenuation correction were reconstructed with a B19f convolution kernel and a slice thickness of $5.0 \mathrm{~mm}$, whereas CT images for anatomic reference were reconstructed with a B31f convolution kernel and a slice thickness of $3.0 \mathrm{~mm}$.

\section{Image Analysis}

${ }^{18}$ F-FDG PET and the CT images were analyzed using the Inveon Research Workplace 4.1 Software (Preclinical Solutions, Siemens Medical Solutions USA). To quantify the mismatch between lesions in the PET and CT images, 2 methods were used: measurement of the distance between the centroids of the PET and CT lesions $(16,17)$ and calculation of the Jaccard similarity coefficient (JSC). The JSC quantifies the spatial overlap of 2 volumes and can be calculated using Equation 1 (19).

$$
\mathrm{J}\left(\mathrm{VOI}_{\mathrm{CT}}, \mathrm{VOI}_{\mathrm{PET}}\right)=\frac{\mathrm{VOI}_{\mathrm{CT}} \cap \mathrm{VOI}_{\mathrm{PET}}}{\mathrm{VOI}_{\mathrm{CT}} \cup \mathrm{VOI}_{\mathrm{PET}}}
$$

Here the volumes of interest on the PET images were delineated using a fixed-threshold region-growing segmentation algorithm. The threshold was set to $40 \%$ of the maximum SUV $(13,16,20)$. The lesions were manually delineated on the low-dose CT images using the lung setting (window center, $-450 \mathrm{HU}$; width, 1,500 HU); these were delineated by an imaging scientist. The lesions on the low-dose CT were sometimes difficult to delineate; in these cases additional imaging was used (contrast CT scan), with an expert nuclear medicine physician supervising.

It has been well established that the motion of structures within the thorax is typically dependent on anatomic location (21). Therefore, it is expected that the effects of respiratory gating also demonstrate such an anatomic dependency. To determine the effect of anatomic location on mismatch between PET and CT scans, lesions were grouped according to their anatomic location within the lungs. These 3 locations are the upper lobes, middle and lower lobes, and lung hilum as described by Grootjans et al. (13). Lesions that demonstrated invasive growth into or attachment to large structures (i.e., the main bronchi, arteries, and veins) of the lung hilum were assigned to the central group.

\section{Statistics}

Statistical analysis was performed using the Mann-Whitney $U$ test. Statistical significance was defined for a $P$ value of less than 0.05 .

\section{RESULTS}

Visual assessment of the images revealed that there was a clear spatial mismatch between the PET and CT images in the FB group. Figure 1 depicts a PET/CT image that exhibits such a spatial mismatch.

The distance between the centroids in the upper lobes and the central region appeared smaller for the FB group, whereas for the $\mathrm{BH}$ group the distance was smaller in the middle and lower lobes. However, these differences were not statistically significant. The results of the distance of the centroids between the PET and CT images are described in Table 3. In Table 4, the results of the JSC are described.

The results show that there is no statistically significant difference in the calculated JSC between the upper and middle

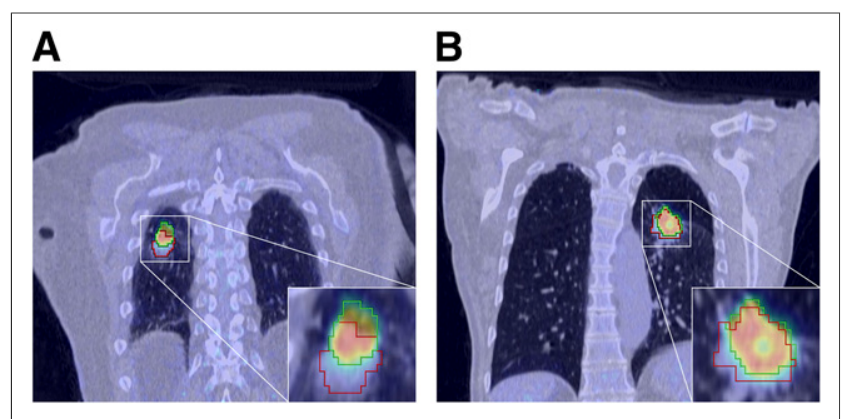

FIGURE 1. Coronal respiratory-gated PET images. (A) Patient with squamous cell carcinoma in FB group. Lesion delineation using PET (green) and CT (red). Difference between centroids of PET and CT images is $10.5 \mathrm{~mm}$. JSC is 0.29 . (B) Patient with adenocarcinoma in $\mathrm{BH}$ group. PET lesion delineation is green, and CT delineation is red. Difference between centroids of PET and CT images is $4.0 \mathrm{~mm}$; JSC is 0.50 .

and lower lobes. However, there is a statistically significant difference between both groups in the lesions located near the lung hilum.

No explanation was found for the outliers as shown in Figures 2 and 3. These lesions were therefore not excluded from the analysis. The results of the distance between the centroids of the PET and CT are shown in Figure 2.

In Figure 3, the results of the JSC are shown.

In the $\mathrm{FB}$ and $\mathrm{BH}$ groups, $9.3 \%$ and $15 \%$ of the lesions, respectively, had no overlap on the PET and CT images. The 7 patients who could not comply with the respiratory instruction and who were subsequently assigned to the FB group did not lead to an inhomogeneous distribution of the 2 groups, because exclusion of these 7 patients did not change the results (data not shown).

\section{DISCUSSION}

This study shows that breathing instructions during the CT scan have no significant impact on the spatial alignment of respiratory-gated PET with CT images. There are differences in the spatial match of the lesions on PET and CT between different anatomic locations of the lesions, but these do not reach statistical significance. Providing breathing instructions during $\mathrm{CT}$ results in a significant difference in spatial match between PET and CT images of lesions in the lung hilum (Fig. 3). However, no definite conclusions can be drawn, because of the limited number of lesions in the lung hilum in the $\mathrm{BH}$ group (3 lesions).

TABLE 3

Results of Distance of Centroids Between PET and CT Images

\begin{tabular}{lccc}
\hline \multicolumn{1}{c}{ Location } & $\begin{array}{c}\text { FB group } \\
(\mathrm{mm})\end{array}$ & $\begin{array}{c}\mathrm{BH} \text { group } \\
(\mathrm{mm})\end{array}$ & $P$ \\
\hline Upper lobes & $4.7 \pm 3.1$ & $6.0 \pm 3.0$ & 0.11 \\
Middle and lower lobes & $5.8 \pm 4.3$ & $5.1 \pm 2.9$ & 0.70 \\
Central & $4.8 \pm 4.6$ & $5.6 \pm 2.0$ & 0.24 \\
Total & $5.1 \pm 3.8$ & $5.5 \pm 2.9$ & 0.16 \\
\hline
\end{tabular}


TABLE 4

Results of JSC

\begin{tabular}{lccc}
\hline \multicolumn{1}{c}{ Location } & FB group & BH group & $P$ \\
\hline Upper lobes & $0.28 \pm 0.17$ & $0.28 \pm 0.19$ & 0.83 \\
Middle and lower lobes & $0.22 \pm 0.16$ & $0.28 \pm 0.18$ & 0.20 \\
Central & $0.39 \pm 0.17$ & $0.13 \pm 0.04$ & 0.04 \\
Total & $0.27 \pm 0.17$ & $0.27 \pm 0.18$ & 0.95 \\
\hline
\end{tabular}

Theoretically, a perfect spatial match could be achieved when a BH CT image is acquired at the same respiratory phase as the gated PET images. However, in practice, this is dependent on the patient's capability to correctly execute the instructions, on the accuracy of the instructions provided by the technologist, and on the interpretation of these instructions by the patient (22).

For this research, patients were assigned to 2 groups: 1 group received a breathing instruction during the CT scan, and 1 group did not. A more accurate way to compare the FB CT to the BH CT protocol would have been an intrapatient comparison by performing both techniques on the same patient. A better effect of the matching between PET and CT on SUV could have been given when the 2 scans were compared. However, it was decided not to perform such a study design after ethical consideration and for reasons of radiation protection.

The optimal gate for the PET scans is usually during the end-expiratory plateau of the respiratory curve. Therefore, to find the best match between the PET and CT scans, patients need to exhale during the CT scan. This is a more difficult maneuver than when a patient is instructed to inhale, which is usually performed during diagnostic CT imaging. In this study, respiratory gating was performed in patients with

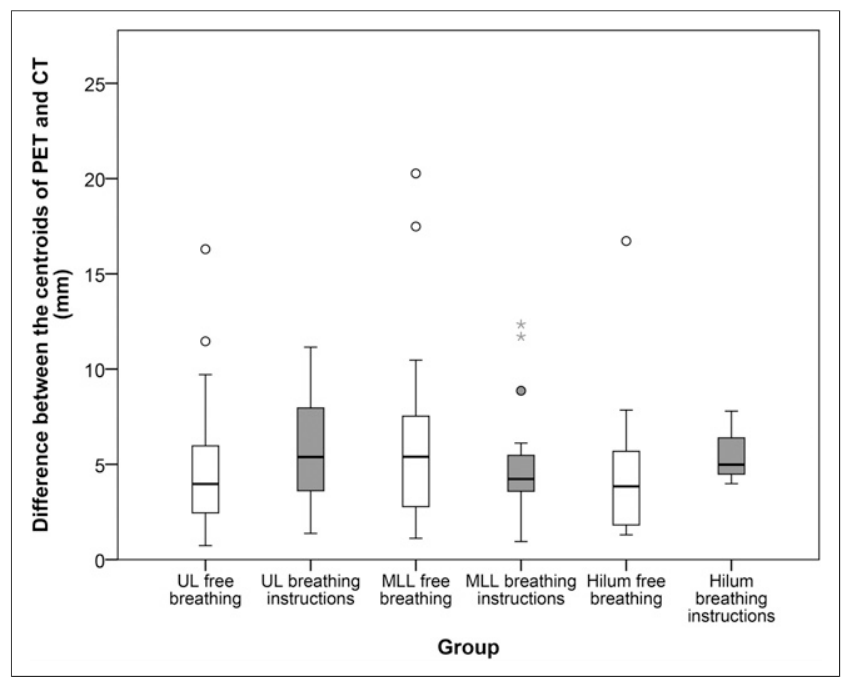

FIGURE 2. Distribution between difference of centroids between PET and CT scan. There are several outliers, depicted as $\bigcirc$, which are values that do not fall in inner fences, and even extreme outliers (*), which are more than 3 times height of boxes. $\mathrm{UL}=$ upper lobes; $\mathrm{MLL}=$ middle and lower lobes; hilum = central lesions, connected to hilum of lung.

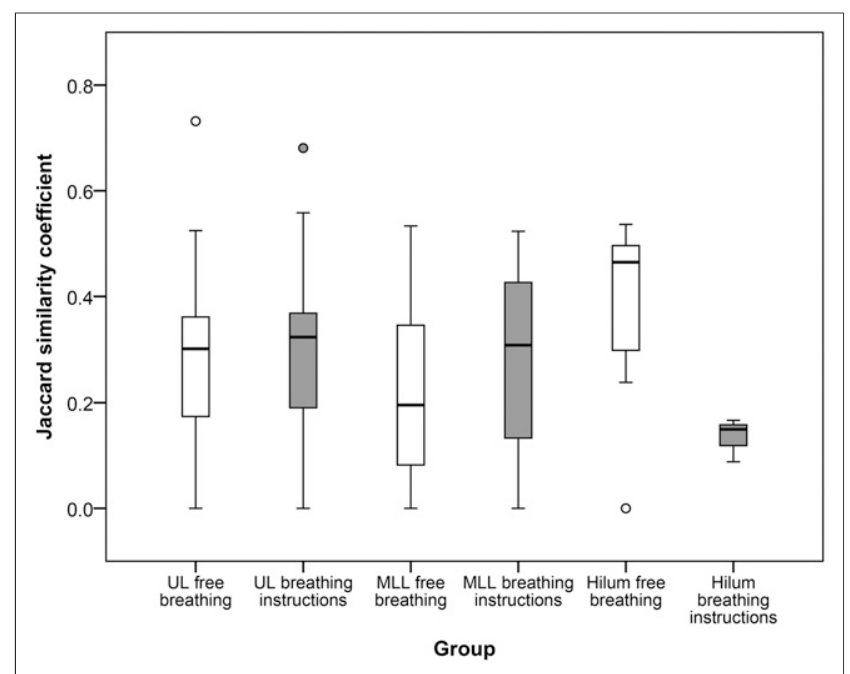

FIGURE 3. Distribution of JSC for both FB and BH groups for 3 localizations. There are several outliers (values that do not fall in inner fences). UL = upper lobes; MLL = middle and lower lobes; hilum $=$ central lesions, connected to hilum of lung.

suspected lung lesions, some of whom experienced respiratory difficulties, such as dyspnoea. Therefore, complying with the provided respiratory instructions is especially strenuous for these patients, as illustrated by the 7 patients who could not comply with the respiratory instruction.

In previous studies, a significantly better match between the PET and the CT images was found when using BH CT $(16,17,19)$. During these approaches, the respiratory signal was measured during the BH CT, and respiratory amplitude during the BH CT was matched to the respiratory amplitude of the PET scan or vice versa. In our approach, the goal was to improve the respiratory-gated PET images. Therefore, the correction was performed the other way around, adjusting the CT images to the optimal gate of the PET. In the present study, a correction method was used, which has a relatively low patient burden, is feasible in clinics with a high patient throughput, and can be implemented without any additional hardware or software. The results of our study imply that most BH CT scans were acquired during a forced deep-expiration and not during rest-expiration. The instructions provided by the operator and the interpretation by the patient can have consequences on the matching between the PET and the CT scan.

There are other techniques to match the CT scan with the optimal gate of the PET scan. These methods, such as respiratory-triggered or -gated $\mathrm{CT}$ acquisitions, may improve spatial matching between PET and CT. However, full CT gating will inevitably increase radiation dose to the patient, compared with standard low-dose CT acquisitions (23). Fortunately, dose-reduction strategies in CT imaging are an area of active research (24). These strategies include modulation of x-ray tube voltage (25) in addition to modulation of x-ray tube current and employment of iterative CT reconstructions (26) to reduce radiation dose to the patient. We are currently investigating the feasibility of respiratory-triggered CT 
acquisitions in combination with these dose-reduction strategies to improve spatial matching of optimal respiratory-gated PET with CT images.

\section{CONCLUSION}

Providing breathing instructions to the patients during the $\mathrm{CT}$ acquisition did not improve the spatial alignment between the PET and CT images. The difficulties experienced in using this clinical protocol, such as patient compliance and operator dependence, emphasize the need for other strategies to improve spatial matching between PET and CT. Without other such strategies, FB CT is the preferred acquisition protocol.

\section{DISCLOSURE}

This study was partially funded by an educational grant from Siemens Healthcare, The Hague, The Netherlands. No other potential conflict of interest relevant to this article was reported.

\section{REFERENCES}

1. Fletcher JW, Djulbegovic B, Soares HP, et al. Recommendations on the use of ${ }^{18}$ F-FDG PET in oncology. J Nucl Med. 2008;49:480-508.

2. Bomanji JB, Costa DC, Ell PJ. Clinical role of positron emission tomography in oncology. Lancet Oncol. 2001;2:157-164.

3. Berghmans T, Dusart M, Paesmans M, et al. Primary tumor standardized uptake value (SUVmax) measured on fluorodeoxyglucose positron emission tomography (FDG-PET) is of prognostic value for survival in non-small cell lung cancer (NSCLC): a systematic review and meta-analysis (MA) by the European Lung Cancer Working Party for the IASLC Lung Cancer Staging Project. J Thorac Oncol. 2008;3:6-12.

4. Zaizen Y, Azuma K, Kurata S, et al. Prognostic significance of total lesion glycolysis in patients with advanced non-small cell lung cancer receiving chemotherapy. Eur J Radiol. 2012;81:4179-4184.

5. Chen HH, Chiu NT, Su WC, Guo HR, Lee BF. Prognostic value of whole-body total lesion glycolysis at pretreatment FDG PET/CT in non-small cell lung cancer. Radiology. 2012;264:559-566.

6. de Geus-Oei LF, van der Heijden HF, Visser EP, et al. Chemotherapy response evaluation with ${ }^{18} \mathrm{~F}$-FDG PET in patients with non-small cell lung cancer. $\mathrm{J} \mathrm{Nucl}$ Med. 2007;48:1592-1598.

7. de Geus-Oei LF, van Laarhoven HW, Visser EP, et al. Chemotherapy response evaluation with FDG-PET in patients with colorectal cancer. Ann Oncol. 2008; 19:348-352.
8. Mikhaeel NG, Hutchings M, Fields PA, O'Doherty MJ, Timothy AR. FDG-PET after two to three cycles of chemotherapy predicts progression-free and overall survival in high-grade non-Hodgkin lymphoma. Ann Oncol. 2005;16:1514-1523.

9. Dose Schwarz J, Bader M, Jenicke L, Hemminger G, Janicke F, Avril N. Early prediction of response to chemotherapy in metastatic breast cancer using sequential ${ }^{18}$ F-FDG PET. $J$ Nucl Med. 2005;46:1144-1150.

10. Callahan J, Binns D, Dunn L, Kron T. Motion effects on SUV and lesion volume in 3D and 4D PET scanning. Australas Phys Eng Sci Med. 2011;34:489-495.

11. Chang G, Chang T, Pan T, Clark JW Jr, Mawlawi OR. Implementation of an automated respiratory amplitude gating technique for PET/CT: clinical evaluation. J Nucl Med. 2010;51:16-24.

12. van Elmpt W, Hamill J, Jones J, De Ruysscher D, Lambin P, Ollers M. Optimal gating compared to 3D and 4D PET reconstruction for characterization of lung tumours. Eur J Nucl Med Mol Imaging. 2011;38:843-855.

13. Grootjans W, de Geus-Oei LF, Meeuwis APW, et al. Amplitude-based optimal respiratory gating in positron emission tomography in patients with primary lung cancer. Eur Radiol. August 6, 2014 [Epub ahead of print].

14. Nehmeh SA, Erdi YE. Respiratory motion in positron emission tomography/ computed tomography: a review. Semin Nucl Med. 2008;38:167-176.

15. Goerres GW, Kamel E, Heidelberg TN, Schwitter MR, Burger C, von Schulthess GK. PET-CT image co-registration in the thorax: influence of respiration. Eur J Nucl Med Mol Imaging. 2002;29:351-360.

16. Fin L, Daouk J, Morvan J, et al. Initial clinical results for breath-hold CT-based processing of respiratory-gated PET acquisitions. Eur J Nucl Med Mol Imaging. 2008;35:1971-1980.

17. Nehmeh SA, Erdi YE, Meirelles GS, et al. Deep-inspiration breath-hold PET/CT of the thorax. J Nucl Med. 2007;48:22-26.

18. Meirelles GS, Erdi YE, Nehmeh SA, et al. Deep-inspiration breath-hold PET/ CT: clinical findings with a new technique for detection and characterization of thoracic lesions. J Nucl Med. 2007;48:712-719.

19. Daouk J, Fin L, Bailly P, Meyer ME. Improved attenuation correction via appropriate selection of respiratory-correlated PET data. Comput Methods Programs Biomed. 2008;92:90-98.

20. Erdi YE, Mawlawi O, Larson SM, et al. Segmentation of lung lesion volume by adaptive positron emission tomography image thresholding. Cancer. 1997;80(12, suppl): 2505-2509.

21. Korreman SS. Motion in radiotherapy: photon therapy. Phys Med Biol. 2012;57: R161-R191.

22. Keall PJ, Mageras GS, Balter JM, et al. The management of respiratory motion in radiation oncology report of AAPM Task Group 76. Med Phys. 2006;33:3874-3900.

23. Daou D. Respiratory motion handling is mandatory to accomplish the highresolution PET destiny. Eur J Nucl Med Mol Imaging. 2008;35:1961-1970.

24. McCollough CH, Bruesewitz MR, Kofler JM Jr. CT dose reduction and dose management tools: overview of available options. Radiographics. 2006;26:503-512.

25. Winklehner A, Goetti R, Baumueller S, et al. Automated attenuation-based tube potential selection for thoracoabdominal computed tomography angiography: improved dose effectiveness. Invest Radiol. 2011;46:767-773.

26. Kalra MK, Woisetschlager M, Dahlstrom N, et al. Radiation dose reduction with sinogram affirmed iterative reconstruction technique for abdominal computed tomography. J Comput Assist Tomogr. 2012;36:339-346. 